\title{
Fractional Lévy motion through path integrals
}

\author{
I. Calvo'ț, R. Sánchez ${ }^{2}$, and B. A. Carreras ${ }^{3}$ \\ ${ }^{1}$ Laboratorio Nacional de Fusión, Asociación EURATOM-CIEMAT, 28040 Madrid, \\ Spain \\ ${ }^{2}$ Fusion Energy Division, Oak Ridge National Laboratory, Oak Ridge, TN 37831, \\ U.S.A. \\ ${ }^{3}$ BACV Solutions Inc., Oak Ridge, TN 37830, U.S.A. \\ E-mail: ivan.calvo@ciemat.es, sanchezferlr@ornl.gov, bacv@comcast.net
}

\begin{abstract}
Fractional Lévy motion (fLm) is the natural generalization of fractional Brownian motion in the context of self-similar stochastic processes and stable probability distributions. In this paper we give an explicit derivation of the propagator of fLm by using path integral methods. The propagators of Brownian motion and fractional Brownian motion are recovered as particular cases. The fractional diffusion equation corresponding to fLm is also obtained.
\end{abstract}

PACS numbers: 02.50.Ey, 05.40.Jc, 05.40.Fb

\section{Introduction}

It is widely known that if $\xi_{2}(t)$ is a Gaussian, uncorrelated noise (i.e. white noise) the Langevin (stochastic) equation

$$
x(t)=x_{0}+\int_{0}^{t} \xi_{2}\left(t^{\prime}\right) \mathrm{d} t^{\prime},
$$

describes ordinary Brownian motion. One of the properties of Brownian motion is that the average squared displacement grows linearly with time, $\left\langle\left(x(t)-x_{0}\right)^{2}\right\rangle \propto t$. However, many transport processes in physical, biological and social systems exhibit anomalous diffusion [1, 2]. That is, $\left\langle\left(x(t)-x_{0}\right)^{2}\right\rangle \propto t^{2 H}$, with $H \neq 1 / 2$, where $H$ is called the Hurst exponent [3]. In the past, several authors have attempted to generalize Eq. (11) in order to accommodate these anomalous processes. The anomalous behaviour may be associated to the existence of spatiotemporal correlations that produce correlated increments $\mathrm{d} x(t):=x(t+\mathrm{d} t)-x(t)$. For that reason, the first proposed generalization had the form

$$
x(t)=x_{0}+\frac{1}{\Gamma(H+1 / 2)} \int_{0}^{t}\left(t-t^{\prime}\right)^{H-1 / 2} \xi_{2}\left(t^{\prime}\right) \mathrm{d} t^{\prime},
$$

known as fractional Brownian motion (fBm) [4], which has been extensively studied and applied [5, 6, 7, 8]. In Eq. (2) the noise $\xi_{2}(t)$ remains Gaussian and uncorrelated $\ddagger$ Corresponding author. 
but, thanks to the convolution with the power-law kernel, the increments $\mathrm{d} x(t)$ become correlated in a way that yields the desired scaling of the mean squared displacement as well as other self-similar properties. In particular, the average motion remains invariant under the transformation $(x, t) \mapsto\left(\lambda^{H} x, \lambda t\right)$, generalizing the self-similarity of the original Brownian motion, which is obviously recovered when $H=1 / 2$ is set in Eq. (2). $H$ is the self-similarity exponent of the process. When rewritten in terms of the Riemann-Liouville fractional integral operators (see Appendix A) fBm reads

$$
x(t)=x_{0}+{ }_{0} D_{t}^{-(H+1 / 2)} \xi_{2} .
$$

The corresponding propagator and diffusion equation of $\mathrm{fBm}$ have been derived in a number of ways in the literature (see, for example, [9] and [10]).

We will devote this paper to the Lévy generalization of fractional Brownian motion, known as fractional Lévy motion (fLm) [11, 12, 13, 14]. The Langevin equation defining the process is

$$
x(t)=x_{0}+{ }_{0} D_{t}^{-H+1 / \alpha-1} \xi_{\alpha},
$$

where $\xi_{\alpha}(t)$ is time-uncorrelated and distributed, for each $t$, according to a symmetric Lévy distribution [15]. We recall here that symmetric Lévy distributions are the symmetric solutions of the Generalized Central Limit Theorem and are parametrized by the stability index, $\alpha \in(0,2]$, and the scale factor, $\sigma>0$. The characteristic function (i.e. the Fourier transform) of a symmetric Lévy distribution $L_{\alpha, \sigma}(u)$ is

$$
\mathcal{F}\left[L_{\alpha, \sigma}\right](k)=\exp \left(-\sigma^{\alpha}|k|^{\alpha}\right) .
$$

In particular, for $\alpha \in(0,2)$, the Lévy distributions have algebraic tails,

$$
L_{\alpha, \sigma}(u) \sim \frac{C_{\alpha}}{|u|^{\alpha+1}}, \quad|u| \rightarrow \infty .
$$

A Lévy distribution with $\alpha=2$ is a Gaussian,

$$
L_{2, \sigma}(u)=\frac{1}{2 \sigma \sqrt{\pi}} \exp \left(-\frac{u^{2}}{4 \sigma^{2}}\right),
$$

and $\sigma$ is related to the second moment, $\left\langle u^{2}\right\rangle=2 \sigma^{2}$.

The need for fLm originates in the observation of power-law (Lévy) statistics for the displacements $\mathrm{d} x(t)$ in many physical systems of interest [1, 2], which is in contrast to the Gaussian character of fBm. In Eq. (41), $H$ is still the self-similarity exponent of the process. It can be shown [15] that finiteness requirements for certain moments of $x$ restrict the admissible values of $H$ to

$$
H \in\left\{\begin{array}{cc}
\left(0, \frac{1}{\alpha}\right] & 0<\alpha \leq 1 \\
(0,1] & 1<\alpha \leq 2 .
\end{array}\right.
$$

Under these circumstances the $s$-th moment of $x$, with $0<s<\alpha$, behaves as $\left\langle|x|^{s}\right\rangle \propto t^{s H}$.

Note that $\mathrm{fBm}$ is recovered from Eq. (41) if $\alpha=2$. Also, the increments $\mathrm{d} x(t)$ of the process are uncorrelated for $H=1 / \alpha$. In this case, ordinary Lévy motion is obtained, of which Brownian motion is a particular case $(H=1 / \alpha=1 / 2)$. In the 
present work we will compute in detail the propagator of fLm through path-integral techniques [16], now familiar in both quantum field theory and statistical physics. Although the form of this propagator has been previously derived in a more abstract way by using self-similarity and stability arguments [11], the path-integral calculation offers a new insight which might help extend the range of applications of fLm and even tackle more complicated situations. Two of us recently computed the propagator of fBm by path integral methods [17] taking advantage of the fact that the measure can be written as the exponential of an action quadratic in the fields. As we will see the fLm path-integral measure is not Gaussian in the fields (except for $\alpha=2$ ) and the computation becomes quite more involved.

The rest of the paper is organized as follows. In Section 2 we construct the appropriate probability measure on the space of realizations of the noise for fLm. The propagator is defined as a particular expectation value. Section 3 gives a detailed calculation of the propagator of fLm in the path integral formalism. In Section 4 the fractional diffusion equation satisfied by the propagator of fLm is worked out. Section 5 contains the conclusions. Appendix A collects some basic definitions on fractional integrals and derivatives.

\section{Construction of the path integral measure}

Assume that the motion of a particle is defined by a stochastic differential equation. The propagator $G\left(x_{T}, T \mid x_{0}, 0\right)$ is, by definition, the probability to find the particle at $x=x_{T}$ at time $t=T$ if initially, $t=0$, it was located at $x=x_{0}$. As mentioned above, the main objective of this paper is to compute the propagator associated to Eq. (4) (we drop the subscript $\alpha$ of $\xi$ from now on) by means of path integrals. Consider trajectories $x(t):[0, T] \rightarrow \mathbb{R}$ with boundary conditions $x(0)=x_{0}$ and $x(T)=x_{T}$. From Eq. (4) we immediately deduce that the boundary conditions of $x(t)$ are translated into the following constraint on $\xi(t)$ :

$$
{ }_{0} D_{T}^{-H+1 / \alpha-1} \xi=x_{T}-x_{0} .
$$

The essential object in the path integral formalism is the probability measure $\mathcal{P}(\xi(t)) \mathcal{D} \xi(t)$ on the space of realizations of the noise, i.e. on the space of maps $\xi(t):[0, T] \rightarrow \mathbb{R}$. Once it is constructed, the propagator is defined as the following expectation value:

$$
G\left(x_{T}, T \mid x_{0}, 0\right)=\int \delta\left({ }_{0} D_{T}^{-H+1 / \alpha-1} \xi-\left(x_{T}-x_{0}\right)\right) \mathcal{P}(\xi(t)) \mathcal{D} \xi(t) .
$$

In order to construct the measure associated to the Langevin equation (44) we will discretize the time in $N+1$ points $t_{n}:=n \epsilon, n=0,1, \ldots, N$, with $\epsilon:=T / N$. The continuum limit, $N \rightarrow \infty$, will be taken eventually. Each path is discretized according to $x_{n}:=x\left(t_{n}\right)$. The appropriate discretization of the noise is made by taking $\xi\left(t_{n}\right)=\epsilon^{-1+1 / \alpha} \xi_{n}$, where each $\xi_{n}$ is an independent random variable distributed according to a symmetric Lévy distribution of index $\alpha$. The factor $\epsilon^{-1+1 / \alpha}$ ensures the 
correct time-dependence of the finite moments of $x,\left\langle|x|^{s}\right\rangle \propto t^{s H}, 0<s<\alpha$. Therefore, the probability measure is naturally defined as

$$
\mathcal{P}(\xi(t)) \mathcal{D} \xi(t)=\prod_{n=1}^{N} L_{\alpha, \sigma}\left(\xi_{n}\right) \mathrm{d} \xi_{n} .
$$

Using the definition of the fractional integral, Eq. (A.1), the constraint (9) can be written as

$$
\frac{1}{\Gamma(H-1 / \alpha+1)} \int_{0}^{T}(T-\tau)^{H-1 / \alpha} \xi(\tau) \mathrm{d} \tau=x_{T}-x_{0}
$$

Discretizing as prescribed above:

$$
\begin{aligned}
\int_{0}^{T}(T-\tau)^{H-1 / \alpha} \xi(\tau) \mathrm{d} \tau & =\sum_{n=1}^{N} \xi(n \epsilon) \int_{(n-1) \epsilon}^{n \epsilon}(T-\tau)^{H-1 / \alpha} \mathrm{d} \tau \\
& =\frac{\epsilon^{H}}{H-1 / \alpha+1} \sum_{n=1}^{N} \xi_{n}\left[(N-n+1)^{H-1 / \alpha+1}-(N-n)^{H-1 / \alpha+1}\right]
\end{aligned}
$$

and we can easily solve for $\xi_{N}$ in terms of $\xi_{n}, n=1, \ldots, N-1$ :

$$
\xi_{N}=A-\sum_{n=1}^{N-1} B_{n} \xi_{n}
$$

with

$$
\begin{aligned}
A & :=\frac{\Gamma(H-1 / \alpha+2)}{\epsilon^{H}}\left(x_{T}-x_{0}\right), \\
B_{n} & :=(N-n+1)^{H-1 / \alpha+1}-(N-n)^{H-1 / \alpha+1}, \quad n=1, \ldots, N-1 .
\end{aligned}
$$

Now, we are ready to write an explicit expression for the expectation value defining the propagator (10). Namely,

$G\left(x_{T}, T \mid x_{0}, 0\right)=\lim _{N \rightarrow \infty} f(T, N) \int \delta\left(\xi_{N}-A+\sum_{n=1}^{N-1} B_{n} \xi_{n}\right) \prod_{n=1}^{N} L_{\alpha, \sigma}\left(\xi_{n}\right) \mathrm{d} \xi_{n}$,

where $f(T, N)$ is a normalization factor which will be determined at the end of the calculation. Equivalently, integrating over $\xi_{N}$ in Eq. (16):

$G\left(x_{T}, T \mid x_{0}, 0\right)=\lim _{N \rightarrow \infty} f(T, N) \int L_{\alpha, \sigma}\left(A-\sum_{n=1}^{N-1} B_{n} \xi_{n}\right) \prod_{n=1}^{N-1} L_{\alpha, \sigma}\left(\xi_{n}\right) \mathrm{d} \xi_{n}$.

It is instructive to show that the path integral of ordinary Brownian motion, usually introduced in a different fashion, coincides with Eq. (16) when $H=1 / \alpha=1 / 2$. The Langevin equation for Brownian motion is (recall Eq. (11))

$$
\dot{x}(t)=\xi_{2}(t) .
$$

The propagator is customarily introduced as

$G\left(x_{T}, T \mid x_{0}, 0\right)=\int \delta\left(x(0)-x_{0}\right) \delta\left(x(T)-x_{T}\right) \exp \left(-\frac{1}{4 \sigma^{2}} \int_{0}^{T} \dot{x}(t)^{2} \mathrm{~d} t\right) \mathcal{D} x(t)$, 
where the paths are weighted by the classical action of the free particle. Now, one can choose the velocity, $v(t)=\dot{x}(t)$, as the integration variable. The transformation is linear and the Jacobian does not depend on the fields. The boundary conditions are simply translated into

$$
\int_{0}^{T} v(t) \mathrm{d} t=x_{T}-x_{0}
$$

Therefore, we can write

$G\left(x_{T}, T \mid x_{0}, 0\right)=\int \delta\left(\int_{0}^{T} v(t) \mathrm{d} t-\left(x_{T}-x_{0}\right)\right) \exp \left(-\frac{1}{4 \sigma^{2}} \int_{0}^{T} v(t)^{2} \mathrm{~d} t\right) \mathcal{D} v(t)$.

If we discretize the paths as above we get

$G\left(x_{T}, T \mid x_{0}, 0\right)=\lim _{N \rightarrow \infty} f(T, N) \int \delta\left(\epsilon \sum_{n=1}^{N} v_{n}-\left(x_{T}-x_{0}\right)\right) \prod_{n=1}^{N} \exp \left(-\frac{\epsilon}{4 \sigma^{2}} v_{n}^{2}\right) \mathrm{d} v_{n}$,

where $f(T, N)$ is a normalization factor. Finally, with a last change of variables, $\xi_{n}:=\epsilon^{1 / 2} v_{n}$ (redefine $f(T, N)$ as needed):

$G\left(x_{T}, T \mid x_{0}, 0\right)=\lim _{N \rightarrow \infty} f(T, N) \int \delta\left(\sum_{n=1}^{N} \xi_{n}-\frac{x_{T}-x_{0}}{\epsilon^{1 / 2}}\right) \prod_{n=1}^{N} \exp \left(-\frac{1}{4 \sigma^{2}} \xi_{n}^{2}\right) \mathrm{d} \xi_{n}$,

which is exactly Eq. (16) for $H=1 / \alpha=1 / 2$ (recall Eq. (17)).

\section{Path integral computation of the propagator of fractional Lévy motion}

The computation of (17) is performed by repeated use of the identity

$$
\int_{-\infty}^{\infty} L_{\alpha, \sigma}(x) L_{\alpha, \sigma}(y-\lambda x) \mathrm{d} x=\left(1+\lambda^{\alpha}\right)^{-1 / \alpha} L_{\alpha, \sigma}\left(\frac{y}{\left(1+\lambda^{\alpha}\right)^{1 / \alpha}}\right), \forall y \in \mathbb{R},
$$

where $L_{\alpha, \sigma}(x)$ is a symmetric Lévy distribution with index $\alpha$ and scale factor $\sigma$, and $\lambda$ is a positive real number. The proof is straightforward. Define $\bar{L}_{\alpha, \sigma}(x):=L_{\alpha, \sigma}(\lambda x)$. Then,

$$
\int_{-\infty}^{\infty} L_{\alpha, \sigma}(x) L_{\alpha, \sigma}(y-\lambda x) \mathrm{d} x=\mathcal{F}^{-1}\left[\mathcal{F}\left[L_{\alpha, \sigma}\right] \mathcal{F}\left[\bar{L}_{\alpha, \sigma}\right]\right]\left(\lambda^{-1} y\right)
$$

Using that $\mathcal{F}\left[\bar{L}_{\alpha, \sigma}\right](k)=|\lambda|^{-1} \mathcal{F}\left[L_{\alpha, \sigma}\right](k / \lambda)$ and $\mathcal{F}\left[L_{\alpha, \sigma}\right](k)=\exp \left(-\sigma^{\alpha}|k|^{\alpha}\right)$ we get:

$$
\mathcal{F}\left[L_{\alpha, \sigma}\right](k) \mathcal{F}\left[\bar{L}_{\alpha, \sigma}\right](k)=|\lambda|^{-1} \exp \left(-\sigma^{\alpha}\left|\left(1+|\lambda|^{-\alpha}\right)^{1 / \alpha} k\right|^{\alpha}\right) .
$$

And Eq. (24) follows easily.

Let us go back to Eq. (17). Using Eq. (24) we integrate out $\xi_{1}$ :

$$
G\left(x_{T}, T \mid x_{0}, 0\right)=\lim _{N \rightarrow \infty} f(T, N) \int L_{\alpha, \sigma}\left(\frac{A-\sum_{n=2}^{N-1} B_{n} \xi_{n}}{\left(1+B_{1}^{\alpha}\right)^{1 / \alpha}}\right) \prod_{n=2}^{N-1} L_{\alpha, \sigma}\left(\xi_{n}\right) \mathrm{d} \xi_{n} .
$$


Fractional Lévy motion through path integrals

Integration of $\xi_{2}$ yields

$$
\begin{aligned}
G\left(x_{T}, T \mid x_{0}, 0\right) & =\lim _{N \rightarrow \infty} f(T, N) \int L_{\alpha, \sigma}\left(\frac{A-\sum_{n=3}^{N-1} B_{n} \xi_{n}}{\left(1+B_{1}^{\alpha}\right)^{1 / \alpha}\left(1+\frac{B_{2}^{\alpha}}{1+B_{1}^{\alpha}}\right)^{1 / \alpha}}\right) \prod_{n=3}^{N-1} L_{\alpha, \sigma}\left(\xi_{n}\right) \mathrm{d} \xi_{n} \\
& =\lim _{N \rightarrow \infty} f(T, N) \int L_{\alpha, \sigma}\left(\frac{A-\sum_{n=3}^{N-1} B_{n} \xi_{n}}{\left(1+B_{1}^{\alpha}+B_{2}^{\alpha}\right)^{1 / \alpha}}\right) \prod_{n=3}^{N-1} L_{\alpha, \sigma}\left(\xi_{n}\right) \mathrm{d} \xi_{n} .
\end{aligned}
$$

And after $N-1$ integrations:

$$
G\left(x_{T}, T \mid x_{0}, 0\right)=\lim _{N \rightarrow \infty} f(T, N) L_{\alpha, \sigma}\left(\frac{A}{\left(1+\sum_{n=1}^{N-1} B_{n}^{\alpha}\right)^{1 / \alpha}}\right) .
$$

It remains to compute

$$
\begin{aligned}
& \lim _{N \rightarrow \infty} \frac{A}{\left(1+\sum_{n=1}^{N-1} B_{n}^{\alpha}\right)^{1 / \alpha}}=\lim _{N \rightarrow \infty} \Gamma(H-1 / \alpha+2) \frac{x_{T}-x_{0}}{T^{H}} \times \\
& N^{H}\left[1+\sum_{n=1}^{N-1}\left((N-n+1)^{H-1 / \alpha+1}-(N-n)^{H-1 / \alpha+1}\right)^{\alpha}\right]^{-1 / \alpha} .
\end{aligned}
$$

In the sequel, $g(N) \sim h(N)$ will mean that $g(N) / h(N) \rightarrow 1$ when $N \rightarrow \infty$. First observe that

$$
\begin{aligned}
& \sum_{n=1}^{N-1}\left((N-n+1)^{H-1 / \alpha+1}-(N-n)^{H-1 / \alpha+1}\right)^{\alpha} \sim \\
& (H-1 / \alpha+1)^{\alpha} N^{\alpha H-1} \sum_{n=1}^{N-1}\left(1-\frac{n}{N}\right)^{\alpha H-1},
\end{aligned}
$$

where we have used

$$
\left(1-\frac{n}{N}+\frac{1}{N}\right)^{H-1 / \alpha+1} \sim\left(1-\frac{n}{N}\right)^{H-1 / \alpha+1}+\frac{H-1 / \alpha+1}{N}\left(1-\frac{n}{N}\right)^{H-1 / \alpha} .
$$

Now, we note that

$$
\sum_{n=1}^{N-1}\left(1-\frac{n}{N}\right)^{\alpha H-1} \frac{1}{N} \sim \int_{1 / N}^{1-1 / N}(1-u)^{\alpha H-1} \mathrm{~d} u=\frac{1}{\alpha H}\left[\left(1-\frac{1}{N}\right)^{\alpha H}-\frac{1}{N^{\alpha H}}\right]
$$

and we have almost reached our goal. Combining Eqs. (31) and (33) we get

$$
\sum_{n=1}^{N-1}\left((N-n+1)^{H-1 / \alpha+1}-(N-n)^{H-1 / \alpha+1}\right)^{\alpha} \sim \frac{(H-1 / \alpha+1)^{\alpha}}{\alpha H} N^{\alpha H} .
$$

Inserting this in Eq. (30):

$$
\lim _{N \rightarrow \infty} \frac{A}{\left(1+\sum_{n=1}^{N-1} B_{n}^{\alpha}\right)^{1 / \alpha}}=(\alpha H)^{1 / \alpha} \Gamma(H-1 / \alpha+1) \frac{x_{T}-x_{0}}{T^{H}} .
$$

Hence,

$$
G\left(x_{T}, T \mid x_{0}, 0\right)=f(T) L_{\alpha, \sigma}\left((\alpha H)^{1 / \alpha} \Gamma(H-1 / \alpha+1) \frac{x_{T}-x_{0}}{T^{H}}\right)
$$


and $f(T)$ can be determined by normalization, $\int_{-\infty}^{\infty} G\left(x_{T}, T \mid x_{0}, 0\right) \mathrm{d} x_{T}=1$ :

$$
f(T)=\frac{(\alpha H)^{1 / \alpha} \Gamma(H-1 / \alpha+1)}{T^{H}},
$$

so that the final expression of the propagator is

$$
G\left(x_{T}, T \mid x_{0}, 0\right)=\frac{(\alpha H)^{1 / \alpha} \Gamma(H-1 / \alpha+1)}{T^{H}} L_{\alpha, \sigma}\left((\alpha H)^{1 / \alpha} \Gamma(H-1 / \alpha+1) \frac{x_{T}-x_{0}}{T^{H}}\right) .
$$

Summarizing, we have obtained that the propagator of fLm is a Lévy distribution depending on the combination $x / t^{H}$, so that the average motion is self-similar with exponent $H$.

\section{Fractional diffusion equation}

For the sake of completeness we derive in this section the fractional diffusion equation which governs the time evolution of the propagator of fLm. Denote by $\hat{G}(k, t)$ the Fourier transform of $G\left(x, t \mid x_{0}, 0\right)$ with respect to $x$. Using Eq. (38), the form of the characteristic function of a Lévy distribution, and the properties of the Fourier transform under rescaling:

$$
\hat{G}(k, t)=\exp \left(-\frac{\sigma^{\alpha} t^{\alpha H}}{(\alpha H) \Gamma^{\alpha}(H-1 / \alpha+1)}|k|^{\alpha}\right) .
$$

Differentiating with respect to $t$ :

$$
\frac{\partial}{\partial t} \hat{G}(k, t)=-\frac{\sigma^{\alpha} t^{\alpha H-1}}{\Gamma^{\alpha}(H-1 / \alpha+1)}|k|^{\alpha} \hat{G}(k, t) .
$$

Fourier inverting, recalling the definition Eq. (A.3) and the identity

$$
\mathcal{F}\left[\frac{\partial^{\alpha} f}{\partial|x|^{\alpha}}\right](k)=-|k|^{\alpha} f(k),
$$

we find:

$$
\frac{\partial}{\partial t} G(x, t)=\frac{\sigma^{\alpha} t^{\alpha H-1}}{\Gamma^{\alpha}(H-1 / \alpha+1)} \frac{\partial^{\alpha}}{\partial|x|^{\alpha}} G(x, t) .
$$

Therefore, the propagator of fLm satisfies a space-fractional diffusion equation with time-dependent diffusivity. Eq. (42) was recently derived by different methods in [18].

The equation for the propagator of fBm (appeared in [10]) is obtained from Eq. (42) in the particular case $\alpha=2$ :

$$
\frac{\partial}{\partial t} G(x, t)=\frac{\sigma^{2} t^{2 H-1}}{\Gamma^{2}(H+1 / 2)} \frac{\partial^{2}}{\partial x^{2}} G(x, t),
$$

which is a diffusion equation with time-dependent diffusivity.

Finally, if $H=1 / \alpha=1 / 2$ we retrieve the standard diffusion equation associated to ordinary Brownian motion:

$$
\frac{\partial}{\partial t} G(x, t)=\sigma^{2} \frac{\partial^{2}}{\partial x^{2}} G(x, t)
$$




\section{Conclusions}

The Langevin equation defining fLm consists of two main ingredients: a timeuncorrelated stochastic noise distributed according to a Lévy distribution and a fractional integral operator which generates the time correlations. In this paper we have derived the propagator of fLm (which was deduced in [11] by using self-similarity arguments) through path integral techniques. That is, we have explicitly constructed a probability measure on the set of realizations of the noise and precisely defined the propagator as an average over this measure space. The computation of the propagator has been performed by discretizing the paths and carefully taking the continuum limit at the end. The fractional diffusion equation associated to fLm has also been derived. We hope that the heuristic power of the path integral formalism will provide new insight on the calculation and help address more complicated cases.

Acknowledgements: I. C. acknowledges the hospitality of Oak Ridge National Laboratory, where this work was carried out. Part of this research was sponsored by the Laboratory Research and Development Program of Oak Ridge National Laboratory, managed by UT-Battelle, LLC, for the US Department of Energy under contract number DE-AC05-00OR22725. B. A. C. acknowledges the hospitality of Laboratorio Nacional de Fusión, Asociación EURATOM-CIEMAT.

\section{Appendix A. Riemann-Liouville fractional integral and differential operators}

The books [19, 20] are excellent introductory texts to fractional calculus containing, in particular, the following definitions.

Assume that $f: \mathbb{R} \rightarrow \mathbb{R}$ is a sufficiently well-behaved function. The RiemannLiouville fractional integral operators of order $\alpha$ are defined as

$$
\begin{aligned}
{ }_{a} D_{x}^{-\alpha} f: & =\frac{1}{\Gamma(\alpha)} \int_{a}^{x}\left(x-x^{\prime}\right)^{\alpha-1} f\left(x^{\prime}\right) \mathrm{d} x^{\prime}, \\
{ }^{b} D_{x}^{-\alpha} f: & =\frac{1}{\Gamma(\alpha)} \int_{x}^{b}\left(x^{\prime}-x\right)^{\alpha-1} f\left(x^{\prime}\right) \mathrm{d} x^{\prime} .
\end{aligned}
$$

As for the Riemann-Liouville fractional differential operators of order $\alpha$, the definition is

$$
\begin{aligned}
{ }_{a} D_{x}^{\alpha} f: & :=\frac{1}{\Gamma(m-\alpha)} \frac{\mathrm{d}^{m}}{\mathrm{~d} x^{m}} \int_{a}^{x} \frac{f\left(x^{\prime}\right)}{\left(x-x^{\prime}\right)^{\alpha-m+1}} \mathrm{~d} x^{\prime}, \\
{ }^{b} D_{x}^{\alpha} f: & =\frac{(-1)^{m}}{\Gamma(m-\alpha)} \frac{\mathrm{d}^{m}}{\mathrm{~d} x^{m}} \int_{x}^{b} \frac{f\left(x^{\prime}\right)}{\left(x^{\prime}-x\right)^{\alpha-m+1}} \mathrm{~d} x^{\prime},
\end{aligned}
$$

where $m$ is the integer number verifying $m-1 \leq \alpha<m$.

Finally, the Riesz fractional differential operator is defined as the symmetric combination

$$
\frac{\partial^{\alpha}}{\partial|x|^{\alpha}}:=\frac{-1}{2 \cos (\pi \alpha / 2)}\left({ }_{-\infty} D_{x}^{\alpha}+{ }^{\infty} D_{x}^{\alpha}\right) .
$$




\section{References}

[1] R. Metzler and J. Klafter. Phys. Reports, 339:1, 2000.

[2] G.M. Zaslavsky. Phys. Reports, 371:461, 2002.

[3] H. E. Hurst. Trans. Am. Soc. Civ. Eng., 116:770, 1951.

[4] B. B. Mandelbrot and J. W. van Ness. SIAM Rev., 10:422, 1968.

[5] F. J. Molz, H. H. Liu, and J. Szulga. Water Resour. Res., 33:2273, 1997.

[6] R. J. Elliott and J. van der Hoek. Fractional Brownian motion and financial modeling. In Trends in Mathematics, Birkhäuser Verlag, Basel, 2001.

[7] R. Sánchez, B. A. Carreras, D. E. Newman, V. E. Lynch, and B. Ph. van Milligen. Phys. Rev. E, 74:016305, 2006.

[8] B. J. Cherayil and P. Biswas. J. Chem. Phys., 99:9230, 1993.

[9] K. L. Sebastian. J. Phys. A: Math. Gen., 28:4305, 1995.

[10] K. G. Wang and C. W. Lung. Phys. Lett. A, 151:119, 1990.

[11] N. Laskin, I. Lambadaris, F. C. Harmantzis, and M. Devetsikiotis. Computer Networks, 40:363, 2002.

[12] T. Huillet. J. Phys. A: Math. Gen., 32:7225, 1999.

[13] S. Painter and L. Paterson. Geophys. Res. Lett., 21:2857, 1994.

[14] S. Painter. Water Resour. Res., 32:1323, 1996.

[15] G. Samorodnitsky and M. S. Taqqu. Stable non-Gaussian processes. Chapman \& Hall, New York, 1994.

[16] R. P. Feynman and A. R. Hibbs. Quantum Mechanics and Path Integrals. McGraw-Hill, New York, 1965.

[17] I. Calvo and R. Sánchez. J. Phys. A: Math. Theor., 41:282002, 2008.

[18] N. W. Watkins, D. Credgington, R. Sánchez, and S. C. Chapman. arXiv:0803.2833.

[19] K. Oldham and J. Spanier. The Fractional Calculus. Academic Press, New York, 1974.

[20] I. Podlubny. Fractional differential equations. Academic Press, New York, 1998. 